\title{
THE POTENTIAL ROLE OF DIETARY FACTORS ON URINARY INCONTINENCE IN THE ELDERLY
}

\author{
E.P. Cherniack, H.F. Lee
}

\begin{abstract}
Maintenance of urinary continence is a complex physiological process. Multiple morbidities can alter this process including polypharmacy, and age-related loss of physiological function. An increasing body of evidence suggests the importance of dietary factors and ingested substances. Modification of nutrients and ingested substances might prove beneficial adjunctive therapies in the treatment of incontinence, but remain unproven. Extrapolation of the results of epidemiologic studies of the relationship of excess caloric intake to continence, from the general adult population, suggests trials of weight loss might help. Population studies of vitamin D supplementation and continence also suggest an association, but prospective experimental trials involving vitamin D supplementation have yet to be done. A potential but far more equivocally documented relationship exists for vitamin B12 and continence. Surprisingly little evidence exists for other potential risk factors for incontinence such alcohol and sweeteners, natural or artificial. Future research should involve prospective trials of weight loss and vitamin D, and exploration of the relationship between other dietary factors and continence.
\end{abstract}

Key words: Urinary incontinence, diet.

\section{Introduction}

Urinary incontinence is a prevalent, persistent health and quality of life problem in the elderly, occurring in 20-40 percent of older adults $(1,2)$. Incontinence has major societal implications, ranking as the second most common medical condition leading to institutionalization. The causes of incontinence are multifactorial, and successful treatment can be challenging. Treatments can include devices (such as those to absorb and retain urine), medications, pelvic floor muscle exercises, surgery, and dietary modification.

Maintenance of urinary continence requires complex neuromuscular coordination, and substances ingested as part of the diet may play an important role. In addition to the role of water, an increasing body of evidence delineates the role of other dietary factors. High caloric intake, fat, acidic foods, vitamin D, vitamin B12, and other nutrients such as calcium, alcohol, zinc, and vitamin $\mathrm{C}$ may also influence urinary continence. The purpose of this manuscript is to describe what is known from published evidence about the relationship of dietary

Division of Geriatrics and Palliative Medicine, University of Miami Miller School of Medicine and the Bruce W. Carter Miami VA Medical Center

Corresponding Author: E. Paul Cherniack, Room CLC 445, Miami VA Medical Center, 1201 NW 16 St., Miami, FL 33125, evan.cherniack@va.gov, 3055757000 ext. 2273

Received February 22, 2017

Accepted for publication March 29, 2017 factors and the pathogenesis of urinary incontinence in the elderly.

\section{Methods}

A literature search was conducted through the PubMed database using such search terms as "urinary incontinence", "nutrients", and "diet", and secondary sources were obtained from the primary sources. Unfortunately, due to the paucity of studies, a systematic review could not be performed.

\section{Excess energy intake (calories, fat)}

A number of studies delineate an association between higher energy intake and greater likelihood of urinary incontinence, including in elderly individuals. Spanish investigators measured anthropomorphic parameters in 471 community-dwelling women who were at least 65 years old (3). Subjects with poorer fitness as manifested by increased body fat percentage, waist circumference, and body mass index, had a higher incidence of urinary incontinence. $(\mathrm{p}<0.05)$ Obese subjects (Body Mass Index $[\mathrm{BMI}>30]$ ) were more likely to suffer from stress incontinence than other types. Unfortunately, this is the only study that attempted to define this association specifically in elderly individuals. 
Other studies including, but not limited to older persons, suggested a relationship between excess body weight, an indirect manifestation of higher caloric intake, and incontinence. In one group of 200 postmenopausal Turkish women, age ranges 47-73, an association existed between the presence of metabolic syndrome and stress incontinence $(p<0.001)(4)$. That group was part of larger investigation in which two hundred other premenopausal women also participated. In the population overall, larger waist circumference also correlated with a greater incidence of stress incontinence $(\mathrm{p}<0.05)$. In a mail survey of 6424 participants from the UK, investigators observed that women at least age 40 with a BMI of over 30 suffered from stress (OR=1.74; 95\% CI 1.22-2.48) or urge (OR 1.46; 95\% CI 1.02-2.09) incontinence more often than normal weight individuals (5). In a second mail survey from the same population, 5816 women, all aged 40 or older, responded to a survey about urinary symptoms and diet (6). The survey ranked amounts of ingested foodstuffs by quintile of intake. Higher quintiles of fat and sugar intake correlated with increased incidence of stress incontinence.

\section{Vitamin D}

While excess macronutrient intake impairs urinary continence, lack of micronutrients may impede continence. Several epidemiologic investigations have outlined a relationship between insufficient vitamin D and incontinence. In one survey, University of Alabama researchers measured 25-hydroxyvitamin D baseline concentrations in 350 community-living adults, half male, mean age of 74 (7). Forty-two months after baseline assessment, investigators observed an association in the cumulative incidence of urge incontinence over the course of the study with vitamin D insufficiency, (defined as $20-30 \mathrm{ng} / \mathrm{ml})(\mathrm{p}=0.03)$. However, statistical analysis revealed no significant association between vitamin $\mathrm{D}$ levels and time to incident urinary incontinence, assessed every six months during the course of the study.

A second epidemiologic study confirmed the relationship between vitamin $\mathrm{D}$ and continence. Turkish investigators at an outpatient geriatrics clinic assessed serum vitamin D levels in 705 patients, $68.2 \%$ female, mean age 72.3 years old (8). An analysis of Vitamin D concentrations noted an inverse relationship between vitamin D sufficiency and incidence of urge incontinence $(p=0.013)$. The researchers suggested these relationships might be the result of vitamin $\mathrm{D}$ action on both smooth and skeletal muscle function.

Other studies that included, but did not focus specifically on an elderly population, also found a relationship between vitamin D levels and UI. In a retrospective cohort study, Parker-Autry et al measured $25-\mathrm{OH}$-vitamin D concentrations in 394 women, average age 62, from a urogynecology clinic population (9). More symptoms of incontinence, as measured by the Incontinence Impact Questionnaire (a seven question quality of life questionnaire), correlated with vitamin D insufficiency $(25-29 \mathrm{ng} / \mathrm{ml})$ in women $(\mathrm{p}<0.001)$. The researchers opined that these findings imply an important role for vitamin D in skeletal muscle efficiency, specifically in the pelvic floor musculature.

Further anecdotal reports outlined a role for vitamin $\mathrm{D}$ in incontinence treatment. At Ohio University, a 78 year old woman with a 6 month history of urge urinary incontinence received 6 months of weekly treatments of $50,000 I U$ of vitamin D2, with complete resolution of her incontinence (10). The patient's initial serum vitamin D concentration of $10 \mathrm{ng} / \mathrm{ml}$ increased to $54 \mathrm{ng} / \mathrm{ml}$ following these treatments. Similarly, at the same institution, $50,000 \mathrm{IU}$ weekly vitamin D2 restored continence in a 59 year old with stress incontinence (10).

\section{Vitamin B12}

Vitamin B12 is another micronutrient with the potential to influence continence. At the University of Pittsburgh, a case-control study of 208 geriatric outpatients 65 years and older examined a potential a link between urinary incontinence and serum vitamin B12 concentrations. The investigation matched cases with incontinence with controls of comparable age, race, sex, cognitive function, genitourinary conditions, medications, and mobility. Subjects with incontinence were 2.63 times more likely to have vitamin B12 deficiency, defined as serum vitamin $\mathrm{B} 12<250 \mathrm{pg} / \mathrm{ml}(\mathrm{p}=0.026)$ than controls. As a causeeffect relationship could not be determined from such a study, the researchers recommended a controlled trial of vitamin B12 supplementation in incontinent patients, which, unfortunately, still remains forthcoming in the two decades since this report (11).

Other studies suggest a more ambiguous relationship between vitamin $\mathrm{B} 12$ and incontinence. In a retrospective analysis conducted at the University of Nebraska geriatrics clinic, researchers investigated the relationship between serum vitamin B12 levels and both urinary and fecal incontinence in 929 elderly outpatients at least 65 years old (12). Covariates which contribute to incontinence including functional status, cognitive status, age, race, gender, medical illnesses, and medications, were also assessed. Although those patients with vitamin B12 deficiency, defined as less than or equal to 300pg/ $\mathrm{ml}$, with both fecal and urinary incontinence were more than two times more likely to have dual fecal and urinary incontinence $(p=0.03)$, no significant relationship was found between vitamin B12 deficiency and isolated urinary incontinence. Unfortunately, this study did not examine the relative severity of incontinence, and failed to examine many other covariates involved in the pathogenesis of incontinence, such as the presence of diabetes, stroke, and prostate diseases (2). Among 119 community-dwelling Canadians, all ages 65-89, investigators did not detect an association between vitamin B12 serum concentrations of less than 165 
omol / L and incontinence (13).

In addition, a prospective cross-sectional study of 119 elderly community-dwelling subjects also presented conflicting evidence. This investigation defined vitamin B12 deficiency as serum B12 <165pg/ml, and assayed serum vitamin B12 and other, more precise markers of deficiency, methylmalonic acid and homocysteine concentrations. No significant relationship existed between urinary incontinence, serum B12 $(p=0.424)$, methylmalonic acid levels $(p=0.386)$, or homocysteine concentrations ( $\mathrm{p}=0.535)$.

\section{Caffeine}

Caffeine would seem to possess a common-sense role in the maintenance of continence. However, in the elderly, caffeine plays a less explicit role based on the published scientific literature. In fact, studies specifically in the elderly remain forthcoming. Therefore, the exact relationship to date can be only inferred from studies in younger individuals, which thus far provide conflicting evidence. In Korea, an epidemiological study noted an association between caffeine and postmenopausal women age 50 and older (14). In this population, including 4,028 women, mean age 63 , a correlation existed between the highest tertile of caffeine intake $(>150 \mathrm{mg} / \mathrm{d})$ and incontinence as determined by both physician $(p=0.012)$ and subject report $(p=0.04)$. A US study gleaned from NHANES data noted a correlation between an intake of at least $204 \mathrm{mg} / \mathrm{d}$, the uppermost quartile, and urinary incontinence (OR=1.47; 95\% C 1.07-2.01) (15). The study also ascertained specific types of incontinence among subjects, and their severity, but observed no relationship between any of these and continence.

\section{Discussion}

Numerous studies explored several dietary factors that might play a role in the pathogenesis and progression of urinary incontinence. Unfortunately, the evidence to date on such dietary factors in urinary incontinence is lacking. The literature is largely based only on epidemiologic studies, leaving much to be investigated in more prospective, controlled trials. Furthermore, most researchers to date have used only subjective measures to quantify incontinence. Importantly, very few studies have investigated these relationships specifically in the elderly. Urinary incontinence significantly impacts the quality of life of the elderly population, and as such, future studies to investigate both possible preventative and curative dietary interventions in the elderly will be crucial.

To date, evidence suggests an association between higher body fat and stress urinary incontinence in the elderly. In addition, studies in younger persons have demonstrated associations between urinary incontinence and metabolic syndrome, BMI $>30$, and high fat and sugar intake, and demonstrated decreased incontinence following weight loss. Researchers have hypothesized that obesity may increase intra-abdominal pressure, leading to increased intra-vesicular pressure and effects on pelvic floor musculature and urethral mobility, worsening both stress and urge urinary incontinence $(3,16,17)$. However, investigation into these associations in the elderly is lacking. Neuromuscular electrophysiological studies of the pelvic floor musculature before and after an intervention might help confirm a role for excess calories in the pathogenesis of incontinence and suggest weight loss as a potential treatment. Future studies might focus on weight loss as a potential treatment specifically in the elderly, who most likely suffer the deleterious effects of incontinence.

Another important but related question that remains to be investigated is whether sugar and sweetener intake is independently associated with urinary incontinence. Sugar and high-glycemic food intake, although known to play a role in weight gain and development of metabolic syndrome, have not been independently studied in relation to urinary incontinence. Many clinicians tout avoidance of even artificial sweeteners and spicy foods to alleviate incontinence, but these await formal trials as to benefit.

Studies in the elderly have demonstrated a consistent association between vitamin D deficiency or insufficiency and urinary incontinence in the elderly. Investigators have proposed that the presence of Vitamin D receptors on the pelvic floor musculature, prostate tissue, and bladder muscle itself suggests a role of vitamin D in function of these structures and maintenance of urinary continence $(7-9,18,19)$. However, future studies should investigate vitamin $\mathrm{D}$ supplementation as a putative treatment for urinary incontinence in the elderly. The evidence on the association between Vitamin B12 and urinary incontinence is conflicting. Further research is needed to determine whether there exists a correlation strong enough to recommend vitamin B12 supplementation for the prevention or treatment of urinary incontinence. However, the benefit of supplementation of either vitamin may require treatment over long periods of time.

The literature, albeit conflicting, suggests a positive correlation between increased caffeine intake and worsening urinary incontinence in younger populations. Further study might be directed at the effect of caffeine in the elderly, in order to determine whether providers should suggest decreased intake for their elderly patients suffering with incontinence. Other dietary factors - including alcohol, calcium, food types, and fiber intake - show weaker possible associations with urinary incontinence.

A more robust response to weight loss might be obtained in future studies combining weight loss with other dietary approaches. Perhaps weight loss together with vitamin D supplementation, for example could prove beneficial in the management of urinary 


\section{incontinence.}

Conflicts of interests: None

\section{References}

1. Milsom I, Abrams P, Cardozo L, Roberts RG, Thuroff J, Wein AJ. How widespread are the symptoms of an overactive bladder and how are they managed? A population-based prevalence study. BJU Int. 2001;87(9):760-6.

2. Collerton J, Davies K, Jagger C, Kingston A, Bond J, Eccles MP, et al. Health and disease in 85 year olds: baseline findings from the Newcastle $85+$ cohort study. BMJ. 2009;339:b4904.

3. Moreno-Vecino B, Arija-Blazquez A, Pedrero-Chamizo R, Alcazar J, GomezCabello A, Perez-Lopez FR, et al. Associations between obesity, physical fitness, and urinary incontinence in non-institutionalized postmenopausal women: The elderly EXERNET multi-center study. Maturitas. 2015;82(2):20814.

4. Otunctemur A, Dursun M, Ozbek E, Sahin S, Besiroglu H, Koklu I, et al Impact of metabolic syndrome on stress urinary incontinence in pre- and postmenopausal women. Int Urol Nephrol. 2014;46(8):1501-5.

5. Dallosso HM, Matthews RJ, McGrother CW, Donaldson MM, Shaw C, Leicestershire MRCISG. The association of diet and other lifestyle factors with the onset of overactive bladder: a longitudinal study in men. Public Health Nutr. 2004;7(7):885-91.

6. Dallosso H, Matthews R, McGrother C, Donaldson M. Diet as a risk factor for the development of stress urinary incontinence: a longitudinal study in women. Eur J Clin Nutr. 2004;58(6):920-6.

7. Vaughan CP, Tangpricha V, Motahar-Ford N, Goode PS, Burgio KL, Allman RM, et al. Vitamin D and incident urinary incontinence in older adults. Eur J Clin Nutr. 2016;70(9):987-9.

8. Kilic MK, Kizilarslanoglu MC, Kara O, Arik G, Varan HD, Kuyumcu ME, et al. Hypovitaminosis D is an independent associated factor of overactive bladder in older adults. Arch Gerontol Geriatr. 2016;65:128-32.
9. Parker-Autry CY, Markland AD, Ballard AC, Downs-Gunn D, Richter HE. Vitamin D status in women with pelvic floor disorder symptoms. Int Urogynecol J. 2012;23(12):1699-705.

10. Gau JT. Urinary incontinence resolved after adequate vitamin D supplementation: a report of two cases. J Am Geriatr Soc. 2010;58(12):2438-9.

11. Rana S, D'Amico F, Merenstein JH. Relationship of vitamin B12 deficiency with incontinence in older people. J Am Geriatr Soc. 1998:46(7):931-2.

12. Endo JO, Chen S, Potter JF, Ranno AE, Asadullah S, Lahiri P. Vitamin B(12) deficiency and incontinence: is there an association? J Gerontol A Biol Sci Med Sci. 2002;57(9):M583-7.

13. Garcia A, Smith M, Freedman M. Vitamin B12 deficiency and incontinence in older people. Can J Urol. 2000;7(4):1077-80.

14. Baek JM, Song JY, Lee SJ, Park EK, Jeung IC, Kim CJ, et al. Caffeine Intake Is Associated with Urinary Incontinence in Korean Postmenopausal Women Results from the Korean National Health and Nutrition Examination Survey. PLoS One. 2016;11(2):e0149311.

15. Gleason JL, Richter HE, Redden DT, Goode PS, Burgio KL, Markland AD. Caffeine and urinary incontinence in US women. Int Urogynecol J. 2013;24(2):295-302.

16. Subak LL, Whitcomb E, Shen H, Saxton J, Vittinghoff E, Brown JS. Weight loss: a novel and effective treatment for urinary incontinence. J Urol. 2005;174(1):190-5.

17. Subak LL, Marinilli Pinto A, Wing RR, Nakagawa S, Kusek JW, Herman WH, et al. Decrease in urinary incontinence management costs in women enrolled in a clinical trial of weight loss to treat urinary incontinence. Obstet Gynecol. 2012;120(2 Pt 1):277-83.

18. Badalian SS, Rosenbaum PF. Vitamin D and pelvic floor disorders in women: results from the National Health and Nutrition Examination Survey. Obstet Gynecol. 2010;115(4):795-803.

19. Vaughan CP, Johnson TM, 2nd, Goode PS, Redden DT, Burgio KL, Markland AD. Vitamin D and lower urinary tract symptoms among US men: results from the 2005-2006 National Health and Nutrition Examination Survey. Urology. 2011;78(6):1292-7. 